\title{
Roxadustat (FG4592) alleviates Radiation-Induced Intestinal Injury by facilitating recovery of intestinal stem cell and reducing damage of intestinal epithelial cell
}

\section{Penglin Xia}

Second Military Medical University

\section{Yajie Yang}

Second Military Medical University

Ruling Liu

Second Military Medical University Haibo Tang

Second Military Medical University

Jicong Du

Second Military Medical University

Ying Cheng

Second Military Medical University

Jianming Cai

Second Military Medical University

Cong Liu

Second Military Medical University

Xiaohua Xu

Tongji University Affiliated Shanghai Pulmonary Hospital

Hu Liu ( $\square$ gzsassliuhu@163.com )

Second Military Medical University

\section{Research}

Keywords: HIF, Radiation-Induced Intestinal Injury (RIII), FG4592, Intestinal Stem Cells (ISCs)

Posted Date: July 8th, 2020

DOl: https://doi.org/10.21203/rs.3.rs-39385/v1

License: (c) (i) This work is licensed under a Creative Commons Attribution 4.0 International License. Read Full License 
Page 2/17 


\section{Abstract}

Background: Radiation induced-intestinal injury (RIII) is a common complication after radiation therapy in patients with pelvic, abdominal or retroperitoneal tumors. The mechanism of RIII includes radiationinduced death of intestinal epithelial cells (IECs) and damage of intestinal stem cells (ISCs), among which damage of ISCs is main cause. Most recently, hypoxia Inducible factor (HIF) has been found to have effect on maintaining stemness and promoting proliferation of ISCs, which suggests a protective role of HIF in the RIII. In this study, we investigated the effect of FG4592, a novel up-regulator of HIF, on the protection of RIII, and further researched its function on ISCs.

Methods: With/without FG4592 treatment, the abdomen of mice was radiated at dose of 25Gy, and then the degree of intestinal injury was assessed by H\&E staining and Brdu label. By intestinal organoid culture, the multiplication capacity and differentiation features of ISCs were detected. Besides, the effects of FG4592 on the radiated IECs were also evaluated by detecting cell viability, apoptosis, and proliferation potential.

Results: FG4592 could effectively up-regulate the expression of HIF-1a and HIF-2a in vivo. An abdominal radiation of 25Gy established the RIII model of mice, by which FG4592 was verified to have protective effect on the intestine from radiation. Morphology and Brdu test of intestinal organoid showed that FG4592 could promote regeneration and differentiation in ISCs after radiation, which were mediated by up-regulating HIF-2 rather than HIF-1.

Conclusion: FG4592, a novel up-regulator of HIF could remit RIII and promote regeneration and differentiation of ISCs after radiation, which were depend on HIF-2 rather than HIF-1.

\section{Introduction}

Radiation induced-intestinal injury (RIII) is a common complication after radiation therapy in patients with pelvic, abdominal, or retroperitoneal tumors. Studies have shown that the incidence of intestinal injury caused by pelvic radiation therapy is about 2-17\% [1]. Currently, it is believed that the mechanism of radioactive intestinal injury mainly includes: on the one hand, radiation induces the death of intestinal epithelial cells [2], the destruction of intestinal barrier [3], and then causes the damage of intestinal tissue; on the other hand, intestinal stem cells are highly sensitive to radiation [4], and a large number of intestinal stem cells die due to radiation so that repair of the epithelial tract is blocked. In recent years, more and more studies have demonstrated that radiation-induced intestinal stem cells (ISCs) damage is a more important causes in the occurrence of radiation intestinal damage and determines the prognosis of patients [5]. Therefore, protection of ISCs from radiation is considered as an effective strategy to prevent and treat RIII [6].

Hypoxia inducible factor (HIF) is a type of transcription factor mainly induced by hypoxia and can subsequently regulate a variety of genes. HIF consists of $a$ and $\beta$ subunits, and contains HIF-1, HIF-2, and HIF-3, among which HIF-1a was most widely studied [7, 8]. Since the discovery of HIF in 1990s, its 
function has been studied extensively. Many studies have shown that HIF plays an important role in hypoxia tolerance [9], tumor proliferation [10], erythropoiesis [11] and so on. Recently, HIF was also reported to plays a key role in the regulation of intestinal stem cell proliferation [12]. These results suggested that up-regulation of HIF may be effective for the prevention and treatment of radiationinduced intestinal injury.

As is known, HIF is regulated by prolyl hydroxylase (PHD) [13]. Under normoxic conditions, HIF-a is hydroxylated by prolyl hydroxylase (PHD) existing in the body. The hydroxylated HIF-a binds to the tumor suppressor protein (vonHippel-Lindau protein, $\mathrm{pVHL}$ ), eventually leading to the degradation of HIF-a. Therefor inhibition of PHD can significantly up-regulate the expression of HIF [14], suggesting a potential protection of targeting PHD on RIII.

In this study, we then investigated the effect of Roxadustat (FG4592), a novel inhibitor to PHD in RIII. As a result, our study demonstrated, for the first time, that FG-4592 could remit the RIII by promoting the recovery of ISCs and inhibiting apoptosis of intestinal epithelial cells post-radiation. Further mechanism research verified that the protective effect of FG-4592 mainly depends on HIF-2 rather than HIF-1. This discovery may provide new therapeutic indications for RIII.

\section{Materials And Methods}

\section{Animals}

Eight-week-old male C57BL/6 mice were purchased from Navy Medical University (Shanghai, China). All protocols were approved by the Navy Medical University (Shanghai, China), in accordance with the U.S. National Institute of Health Guide for Care and Use of Laboratory Animals (NIH Publication No.96 - 01). Establishment of Radiation-Induced intestinal Injury Model of Mice

The mice were immobilized in a circumscribed box in which only the abdomen of mice was exposed to radiation. The other parts of the box were shielded with lead. The abdomen of mice was radiated with a total dose of $25 \mathrm{~Gy}$ at the rate of $1 \mathrm{~Gy} / \mathrm{min}$. The radiation source was $y$ ray ${ }^{60} \mathrm{Co}$ from the Radiation Center (Faculty of Naval Medicine, Navy Medical University).

\section{Cell Culture}

Modek cells were obtained from American Type Culture Collection (ATCC, Gaithersburg, MD), and maintained in RPMI Medium Modified (HyClone) with $10 \%$ fetal bovine serum (gibco) and $1 \%$ penicillinstreptomycin-glutamine (gibco) at $37^{\circ} \mathrm{C}$ in a $5 \% \mathrm{CO}_{2}$ humidified chamber.

\section{Intestinal Organoid Culture}

After the mice were sacrificed by cervical dislocation, and then disinfected with $75 \%$ alcohol, and the abdominal cavity was opened to extract the small intestine tissue of the mouse. Subsequently, the 
intestinal segment is settled by gravity. After the crypts are separated and plated, the culture plate is preheated, and then the appropriate amount of crypts that have been separated is resuspended at a ratio of 1:1 medium (STEMCELL): Matrigel (Corning), and then plated for organoid culture. At designed time points, the morphology of intestinal organoid was observed.

\section{Histopathology and Immunohistochemistry and Immunofluorescence}

The intestinal was primarily fixed with $10 \%$ neutral buffered formalin for $24 \mathrm{~h}$. The tissues were paraffinembedded and sectioned at an average of $5 \mathrm{um}$. These sections were then stained with hematoxylin and eosin(H\&E), and the common pathological morphology was observed. The sections were de-waxed, rehydrated, and incubated in $0.3 \% \mathrm{H}_{2} \mathrm{O}$, to block endogenous peroxidase. Covered antibodies included BRDU (1/200), HIF1(Abcam,1/200), HIF2(Abcam,1/200), OLFM4(Abcam,1/200), Wnt3a(Abcam,1/500), Axin(Abcam,1/500).

\section{Fluorescence in situ hybridization}

After paraffin sections are dewaxed, they are sequentially washed with xylene and different concentrations of alcohol, and then protease is added dropwise for digestion. Subsequent hybridization. After washing with water, DAPI staining solution was added dropwise. After washing, the slides were counted and images were acquired using a fluorescence microscope.

\section{Radiations}

Radiations were performed using a ${ }^{60} \mathrm{Co}$ source at the Radiation Center (Faculty of Naval Medicine, Naval Medical University, China). The mice received abdominal radiation of $25 \mathrm{~Gy}$ at a dose rate of $1 \mathrm{~Gy} / \mathrm{min}$, and Modek cells were radiated with the designated dose.

\section{Western Blot Analysis}

After treatment, Modek cells were collected, resuspended in PBS, and washed three times. They were collected in extraction buffer(1\%TritonTM X-100,0.5\% sodium deoxycholate,20 mM Tris- $\mathrm{HCl}, \mathrm{pH}$ 7.5,12 mM glycerophosphate, $150 \mathrm{mM} \mathrm{NaCl}, 5 \mathrm{mM}$ EGTA,10 mM NaF,3 mM dithiothreitol,1 mM sodium orthovanadate, $1 \mathrm{mM}$ phenyl-methylsulfonyl fluoride and $20 \mathrm{~g} / \mathrm{ml}$ aprotinin) and incubated on ice three times for $10 \mathrm{~min}$. The lysate was centrifuged $\left(12,000 \mathrm{~g}\right.$ for $15 \mathrm{~min}$ at $\left.4^{\circ} \mathrm{C}\right)$ to obtain the supernatant containing the protein. After the mice were treated accordingly, the mice were sacrificed by cervical dislocation and the intestinal tissues were extracted. After washing the intestinal tissue three times with PBS, an appropriate amount of protein lysate was added, and then homogenized with a homogenizer, followed by crushing with an ultrasonic breaker. Centrifuge at $14000 \mathrm{~g}$ with a high-speed centrifuge, take the protein supernatant and add a certain loading buffer, and then $100^{\circ} \mathrm{C}$ for $10 \mathrm{~min}$.

Equal amounts of proteins were separated on a 10\% SDS-PAGE gel and transferred to nitrocellulose membranes. Then, $5 \%$ low-fat milk was used to block the membranes for $2 \mathrm{~h}$, and then the membranes were washed with TBST three times for $10 \mathrm{~min}$. The membranes were probed overnight at $4{ }^{\circ} \mathrm{C}$ with 
antibodies recognizing the following antigens: HIF1a(abcam,1/1000), HIF2a (abcam,1/1000), GAPDH (CST,1/1000). After washing three times with TBST, the corresponding secondary antibody was incubated for 2 hours at room temperature. After TBST was washed 3 times, it was processed on a gel imager (Gene) using an HRP developing solution (thermo).

\section{Statistical analysis}

All experimental data in this study were statistically and analyzed using SPSS19.0. Two independent samples were compared using the $t$ test, and multiple groups were compared using ANOVA. $P<0.05$ indicates statistical difference; correlation analysis uses rank sum test, $P<0.05$ indicates statistical difference.

\section{Results}

\section{FG4592 up-regulates the expression of HIF-1a and HIF-2 $a$ in mouse intestinal tissue}

Firstly, the effect of FG4592 on the expression of HIF-1 and HIF-2 was investigated. FG4592 $(25 \mathrm{mg} / \mathrm{kg})$ was intraperitoneal injected and then the small intestine tissue and colon tissue were taken to extract proteins at 2, 4, 6, 10, 24, and 48 hours after administration. The expressions of HIF-1a and HIF-2a were detected by Western blot. The results showed that after intraperitoneal application of FG4592, HIF-1 $a$ and HIF-2a were up-regulated over time in small intestine (Fig. 1A) and colon tissues (Fig. 1B). However, the expression of HIF-2a was elevated in advance and more obviously than HIF-1a, no matter in small intestine or colon tissues. Further, the results of immunohistochemical also proved that FG4592 could upregulate the expression HIF-1a (Fig. 1C, D) and HIF-2a (Fig. 1E, F) in small intestine, among which HIF-2a reach higher fold of change.

\section{Fg4592 Remits Radiation-induced Intestinal Injury Of Mice}

Next, we determined the effect of FG4592 on the radiation-induced intestinal injury (RIII). 2 hours before radiation the mice were intraperitoneal injected with FG4592 $(25 \mathrm{mg} / \mathrm{kg})$, and then received abdominal radiation of $25 \mathrm{~Gy}$. At 1st, 3rd day post-radiation, the small intestine tissues were taken to assess the degree of injury by H\&E staining and evaluate proliferation ability by Brdu immunofluorescence. Besides, the Lgr5 + fluorescence assay was also performed to detect the mounts of intestinal stem cells (ISCs). As a result, upon receiving radiation, the small intestinal was damaged seriously showing that the length of intestinal villi and the number of crypts decreased, and inflammatory exudation. However, FG4592 effectively remitted above damages (Fig. 2A, B). The status of intestinal stem cells after radiation has an important effect on the regeneration of intestinal epithelial cells. Experimental findings showed that FG4592 improved the reduction of Lgr5 + cells due to radiation-induced injury (Fig. 2C, D), suggesting that FG-4592 may play an important role in promoting intestinal stemness. In addition, we also explored the effect of FG-4592 on cell proliferation by Brdu immunofluorescence and found that radiation can inhibit 
the proliferation of intestinal cells. In contrast, FG-4592 could enhance the Brdu immunofluorescence compared with radiation alone group (Fig. 2E, F).

\section{FG4592 promotes the regeneration and differentiation of intestinal stem cells by activating Wnt/ $\beta$-catenin pathway.}

In previous studies, we found that FG4592 can promote the regeneration of epithelial cells after intestinal radiation in mice, reduce the damage of intestinal crypts, and increase the number of Lgr $5+$ cells, suggesting that FG4592 may has a promoting effect on ISCs. Herein, to further verify this effect, the small intestine organoid culture technology, an important evaluation methodology for proliferation ability of ISCs, was applied. Mice were intraperitoneally injected with FG-4592 in advance $(25 \mathrm{mg} / \mathrm{kg})$ and then received 25 Gy abdominal radiation. 24 hours later, the small intestine crypts were extracted for organoid culture. With culture for 5 days, the morphology of organoid could be observed, and their proliferation abilities were also determined by Edu assay and OLFM4 immunofluorescence staining. The results showed that radiation resulted in decreased surface area and budding rate of intestinal organoids, indicating a suppressive effect on ISCs. However, FG4592 increased the surface area and budding rate post-radiation (Fig. 3A, G). In addition, FG-4592 also elevated the positive rate of Brdu (Fig. 3C, I) and OLFM4 (Fig. 3B, H) fluorescence staining compared with radiation alone group. Except experiments in vivo, we also conducted direct observation of small intestine organoid in vitro. The small intestinal organoids were cultured in advance with or without FG4592, and then received 7 Gy radiation. 3 days later, the morphology of organoid was observed and fluorescence intensity of Brdu and OLFM4 staining were measured. In consistent with results in vivo, FG4592 also increased the surface area and budding rate of organoids (Fig. 3D, J) and raised the fluorescence intensity of Brdu (Fig. 3F, L) and OLFM4 (Fig. 3E, K) staining after radiation.

For small ISCs, the Wnt/ $\beta$-catenin pathway play a critical role in the maintenance of stemness and promotion of proliferation. Therefore, the effect of FG4592 on the Wnt/ $\beta$-catenin pathway was also investigated. Treated with FG4592 $(10 \mu \mathrm{M})$ small intestinal organoids were then collected to examine the expression of Wnt3a, Axin and $\beta$-catenin at different time points. The results demonstrated that FG4592 could directly activate Wnt/ $\beta$-catenin pathway in a time-dependent manner, in which Wnt3a expressed the highest fold change (Fig. 3M).

Above results indicated that FG4592 could alleviate the inhibition of ISCs from radiation and promote their regeneration by activating $\mathrm{Wnt} / \beta$-catenin pathway.

\section{FG4592 protects against radiation-induced intestinal injury by up-regulating HIF-2 rather than HIF-1}

As an inhibitor of PHD, FG4592 could up-regulate the expression of HIF. Since the promoting effect of FG4592 on ISCs was confirmed, we then subsequently investigated if HIF was involved and which subtypes of HIF was decisive. Small intestinal organoids were treated with FG-4592 for 2 hours, and simultaneously the HIF-1 inhibitor, 2-Methoxyestradiol (2-MeOE2) and HIF-2 inhibitor, PT2385 were added. After exposure to $7 \mathrm{~Gy}$ radiation, the morphology of small intestinal organoids was observed. As a result, 
the protective effect of FG4592 on the small intestinal organoids were inhibited by PT2385 rather than 2MeOE2 (Fig. 4A, B), indicating HIF-2 may be decisive. Further investigation to the activation of Wnt/ $\beta$ catenin pathway also verified that PT2385 rather than 2-MeOE2 could significantly block the upregulation of Wnt3a and Axin that were induced by FG4592 (Fig. 4C).

\section{Fg4592 Reduces Damage Of Intestinal Epithelial Cells From Radiation}

Above results have confirmed the protective effect of FG4592 on ISCs. In general, the main causes to radiation-induced intestinal injury rest on inhibition of ISCs and injury of intestinal epithelial cells (IECs). So, we further investigated the effect of FG4592 on the IECs. In this study, the Modek cell, a small intestinal epithelial cell line of mice was utilized. Pretreated with FG4592, Modek was radiated, and then the cell viability, apoptosis and proliferation were detected. As a result, radiation decreased cell viability, induced apoptosis, led to G2/M phase arrest of cell cycle and inhibited proliferation. In comparison, FG4592 pretreatment could obviously increase cell viability (Fig. 5A), reduce apoptosis (Fig. 5B, C), alleviate cell cycle arrest (Fig. 5D, E) and promote proliferation (Fig. 5F, G) in Modek post-radiation. These results indicated that besides promoting regeneration and proliferation of ISC, FG4592 also directly remitted damage of intestinal epithelial cells induced by radiation.

\section{Discussion}

lonizing radiation has virtual clinical significance for the treatment of abdominal malignant tumors. During radiotherapy, the toxicity of ionizing radiation to healthy tissues is still the main side effect that limits its clinical application [15]. Radiation-induced intestinal injury causes intestinal epithelial damage, destruction of the mucosal barrier, and mucosal inflammation in the early stage [16]. Hypoxia-inducible factors (HIF) are important regulators of maintaining intestinal homeostasis. HIF participates in the regulation of many target genes, and then exerts a variety of biological effects. Virtually, FG4592 is a proline hydroxylase inhibitor that mediates the regulation of HIF metabolism [17]. Sun [18] found that upregulating HIF expression in the intestine can reduce intestinal damage induced by ionizing radiation. At the same time, after conditionally knocking out the expression of HIF in the intestine of mice, the radiation damage of the intestine of the mice is further aggravated. Intestinal Epithelial Cells-Derived Hypoxia-Inducible Factor-1 a Is Essential for the Homeostasis of Intestinal Intraepithelial Lymphocytes. The research of HIF in the field of radiation is not limited to radiation-induced intestinal injury. Its research also involves radiation lung injury and tumor radiotherapy sensitization. However, the mechanism of action of HIF on radiation intestinal injury has not been fully elucidated. Taniguchi CM [19] found that DMOG mediates HIF2 a to produce radiation protection for ISC, which further suggests that HIF may have a certain regulatory effect on ISC after radiation.

To verify the effect of FG4592 on the intestine after radiation, in our experimental research, first of all, we found that there were different expressions of HIF1a and HIF2 $a$ in the small intestine and colon of mice 
after FG4592 administration. We speculated that it was due to the difference in the absorption capacity of HIF1a/ 2a in the colon and small intestine. Subsequently, the effect of FG4592 on radiation-induced intestinal injury was tested. Our data found that FG4592 ameliorated the degree of intestinal injury in the small intestine of mice after radiation. It is particularly worth noting that FG4592 could up-regulate the expression of Lgr5 + intestinal stem cells after irradiation. This led to the restoration of intestinal epithelial proliferation ability, which was vital for intestinal injury repair. Therefore, we further used intestinal organoid culture technology as a support to further explore the effect of FG4592 on ISCs. We found that, whether in vivo or in vitro, FG4592 could promote budding of intestinal organoids, which promoted ISC survival after radiation. OLFM4 is an important indicator of Lgr5 + ISC. Detection of OLFM4 immunofluorescence revealed that ionizing radiation had a significant inhibitory effect on Lgr5 + ISC, and FG4592 could significantly alleviate the suppression of OLFM4 after radiation. Lgr5 + ISC has an important effect on the self-proliferation of intestinal stem cells. Our data found that the increased expression of Lgr5 + after radiation can significantly promote the proliferation of intestinal organoids, thereby promoting the filling of intestinal crypts by ISC and the differentiation of ISC into cells of intestinal tissues. To further identify whether FG4592 exerts radiation biological effects through HIF1a or HIF2a. We then used HIF1 and HIF2 inhibitors 2-MeOE2 (HIF1 a inhibitors) and PT2385 (HIF2a inhibitors) to clarify. Our experimental data found that after applying HIF1a inhibitor (2-MeOE2), it was found that the damage to intestinal organoids was not aggravated, but PT2385 (HIF2a inhibitor) could significantly further aggravate the suppression of intestinal organogenesis by radiation. It showed that FG4592 may play a radiation protection effect on ISC through HIF2a. The evidence found that stimulating HIF2a can promote the self-proliferation of liver cells. Hypoxia-inducible factor 2 alpha is essential for hepatic outgrowth and functions via the regulation of leg1 transcription in the zebrafish embryo [20]. More research showed that HIF plays an important role in the mobilization of mesenchymal stem cells. Dimethyloxalyl Glycine Regulates the HIF-1 Signaling Pathway in Mesenchymal Stem Cells.[21] Inhibiting the expression of HIF2a aggravated the damage of radiation to ISCs, which may be related to the suppression of the Wnt pathway after irradiation, but the inhibition of HIF1 a did not show the effect of aggravating ISC damage. At present, there is little article on HIF2 $a$ in radiation intestinal injury, therefore its specific mechanism of action needs to be further studied. Intestinal epithelial cells are important part of the intestinal mucosal barrier. In order to further explore the effect of FG4592 on the radiation sensitivity of intestinal epithelial cells, we used Modek cells as the research object and found that FG4592 could significantly increase the viability of Modek cells after radiation, reduce radiation-induced apoptosis, and alleviate radiation-induced G2M phase block of Modek, which make the cells pass the cycle checkpoint, and then reduced the inhibition of ionizing radiation on the proliferation of Modek cells. Therefore, FG4592 could significantly reduce the radiation sensitivity of Modek cells and mediate its antidamage effect and proliferation repair effect during radiation. It further improved radiation-induced disturbances of the upper intestinal barrier.

In conclusion, our study demonstrated for the first time, that FG4592 could protect ISCs from radiation to remit radiation-induced intestinal damage, which was mediated by HIF-2 rather than HIF-1 (Fig. 6).

\section{Declarations}




\section{Abbreviations}

Radiation induced-intestinal injury (RIII) 『intestinal epithelial cells (IECs) \intestinal stem cells (ISCs)》 hypoxia Inducible factor (HIF) 『prolyl hydroxylase (PHD)『2-Methoxyestradiol (2-MeOE2)

\section{Declarations}

\section{Ethics approval and consent to participate}

All animal experiments conformed to the National Institute of Health Guide for the Care and Use of Laboratory Animals' (NIH Publication No. 85-23, National Academy Press, Washington, DC, revised 1996), with the approval of the Laboratory Animal Center of the Second Military Medical University, Shanghai. The approval ID for this study was 20191512.

\section{Consent for publication}

Written informed consent for publication was obtained from all participants

\section{Availability of data and material}

All data generated or analyzed during this study are included in this published article and its supplementary information files.

\section{Competing interests}

The authors declare that they have no competing interests

\section{Founding}

This work was sponsored by Natural Science Foundation of Shanghai (No. 18ZR1449700), Shanghai Sailing Program (No.18YF1429200) and National Natural Science Foundation of China (No.81972978)

\section{Authors' contributions}

Jianming Cai, Xiaohua Xu, and Hu Liu designed the study. Penglin Xia, Yajie Yang, Ruling Liu, Haibo Tang, Jicong Du performed the experiments. Ying Cheng and Cong Liu analyzed the data. Penglin Xia and Hu Liu wrote the paper, Jianming Cai supported fund assistance. All authors read and approved the final manuscript.

\section{Acknowledgements}

We appreciate the radiation device department of Second Military Medical University, which produced gamma-rays 


\section{References}

1. DM S. G S, L V, PE W, B C. Prevention of chemotherapy and radiation toxicity with glutamine. Cancer treatment reviews. 2003;29(6):501-13.

2. N DLHE. R, N D, A A, S G, et al. Secretion of acid sphingomyelinase and ceramide by endothelial cells contributes to radiation-induced intestinal toxicity. Cancer research. 2020.

3. C S-P MCV, B VMFP. P, V H, et al. PrP(c) deficiency and dasatinib protect mouse intestines against radiation injury by inhibiting of c-Src. Radiotherapy oncology: journal of the European Society for Therapeutic Radiology Oncology. 2016;120(1):175-83.

4. MM ML, BPC GYLJS. C, H G, et al. The vanillin derivative VND3207 protects intestine against radiation injury by modulating p53/NOXA signaling pathway and restoring the balance of gut microbiota. Free Radic Biol Med. 2019;145:223-36.

5. A C-P MY, C P, dIR S, N D. URI is required to maintain intestinal architecture during ionizing radiation. Science (New York, NY). 2019;364(6443).

6. X LWBJL. W, M E, J G, L Z, et al. Inhibition of CDK4/6 protects against radiation-induced intestinal injury in mice. J Clin Investig. 2016;126(11):4076-87.

7. L Z, J C, L D, H L. TiPARP forms nuclear condensates to degrade HIF-1a and suppress tumorigenesis. Proceedings of the National Academy of Sciences of the United States of America. 2020.

8. JW W. D S, M B, M F, S R. Oxygen sensing mechanisms in cells. The FEBS journal. 2020.

9. $\mathrm{H} \mathrm{N}, \mathrm{H} \mathrm{W}, \mathrm{K} \mathrm{J}, \mathrm{X}$ Y. Transcriptome analysis reveals differential immune related genes expression in Ruditapes philippinarum under hypoxia stress: potential HIF and NF-KB crosstalk in immune responses in clam. BMC genomics. 2020;21(1):318.

10. Y L, X S, Y L, B C, W Z, L W, et al. LncRNA BCRT1 promotes breast cancer progression by targeting miR-1303/PTBP3 axis. Molecular cancer. 2020;19(1):85.

11. ML N, EL C, EA PN, SP S. T, R A, et al. Erythropoietin and a hypoxia-inducible factor prolyl hydroxylase inhibitor (HIF-PHDi) lowers FGF23 in a model of chronic kidney disease (CKD). Physiological reports. 2020;8(11):e14434.

12. XQ YC, BY Y, YH T, SH T. T, CH L, et al. Deferoxamine preconditioning activated hypoxia-inducible factor-1 $a$ and MyD88-dependent Toll-like receptor 4 signaling in intestinal stem cells. Journal of pediatric surgery. 2018;53(11):2349-56.

13. K S, Z L, Y Y, X Z. The prolyl hydroxylase inhibitor roxadustat: Paradigm in drug discovery and prospects for clinical application beyond anemia. Drug discovery today. 2020.

14. HY L, Y Y, YH F. Y W, XY G. Hypoxia-inducible factor-1a: A promising therapeutic target for vasculopathy in diabetic retinopathy. Pharmacological research. 2020;159:104924.

15. NK VPSXARWCAL. A, G C, et al. Transient enhancement of p53 activity protects from radiationinduced gastrointestinal toxicity. Proc Natl Acad Sci USA. 2019;116(35):17429-37.

16. SY HJ, YH KSPKK. K, J N, et al. Pravastatin Alleviates Radiation Proctitis by Regulating Thrombomodulin in Irradiated Endothelial Cells. International journal of molecular sciences. 
2020;21(5).

17. JW AS. W, CJ S, R C. Hypoxia-inducible factor (HIF) prolyl hydroxylase inhibitors induce autophagy and have a protective effect in an in-vitro ischaemia model. Scientific reports. 2020;10(1):1597.

18. K Y LSTLHT. Y M, M Y, et al. Intestinal Epithelial Cells-Derived Hypoxia-Inducible Factor-1a Is Essential for the Homeostasis of Intestinal Intraepithelial Lymphocytes. Frontiers in immunology. 2019;10:806.

19. CM T, YR M, AN D, C W, EB R, TF A, et al. PHD inhibition mitigates and protects against radiationinduced gastrointestinal toxicity via HIF2. Science translational medicine. 2014;6(236):236ra64.

20. TY L, CF C, HY C, CY C, JL CHL. W, et al. Hypoxia-inducible factor 2 alpha is essential for hepatic outgrowth and functions via the regulation of leg1 transcription in the zebrafish embryo. PloS one. 2014;9(7):e101980.

21. B Z TG, L Z, L J, L Z, P Y, et al. Dimethyloxalyl Glycine Regulates the HIF-1 Signaling Pathway in Mesenchymal Stem Cells. Stem cell reviews and reports. 2020.

\section{Figures}

$A$

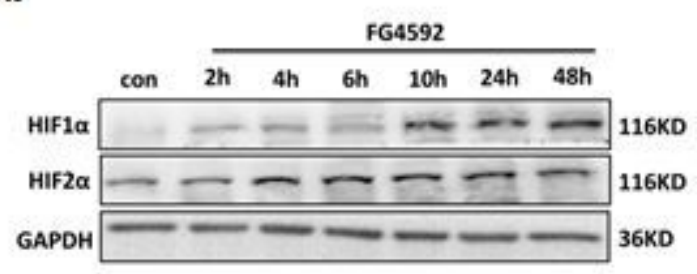

B

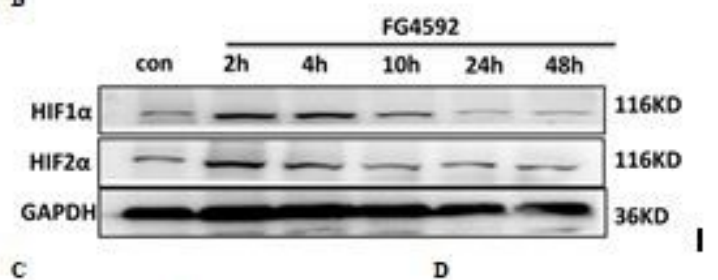

c
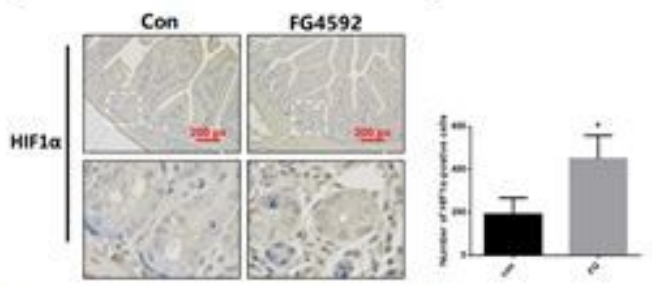

E

F

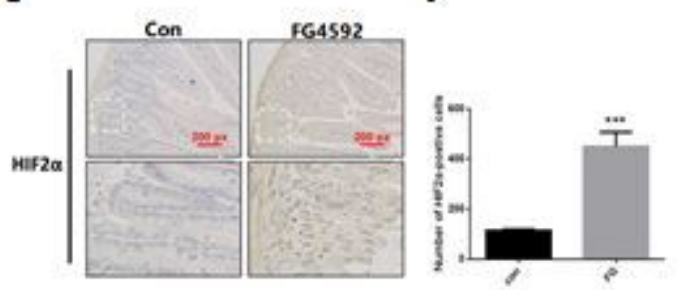

Figure 1 
FG4592 up-regulates the expression of HIF-1 $a$ and HIF-2a in mouse intestinal tissue. Mice were intraperitoneal injected with FG4592 at $25 \mathrm{mg} / \mathrm{kg}$, and then small intestine and colon tissue were taken to extracted proteins at $2 \mathrm{~h}, 4 \mathrm{~h}, 6 \mathrm{~h}, 10 \mathrm{~h}, 24 \mathrm{~h}$, and $48 \mathrm{~h}$. By Western Blot experiments, the expression of HIF-1a, HIF-2a in small intestine (A), and colon tissue (B) were detected. 2 hours after intraperitoneal injected with FG4592, the small intestine tissues of mice were collected to perform immunohistochemistry, by which the HIF-1 $a(C)$ and HIF-2a (E) were stained. (D) and (F) were quantification for the expression of HIF-1a and HIF-2a respectively. The statistical results are expressed by Mean $\pm S D$. * represents $p<0.05$, *** represents $p<0.001$.

A

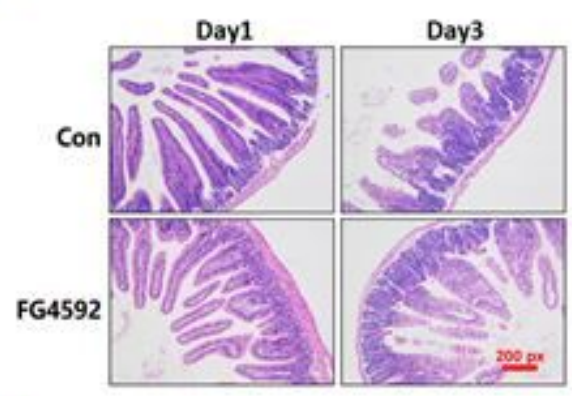

C

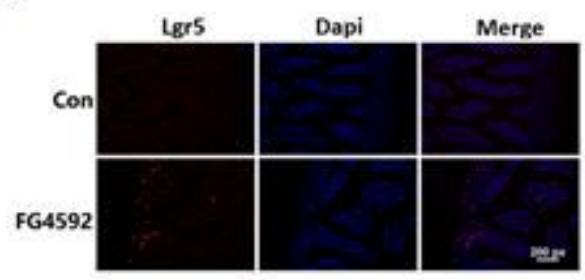

E

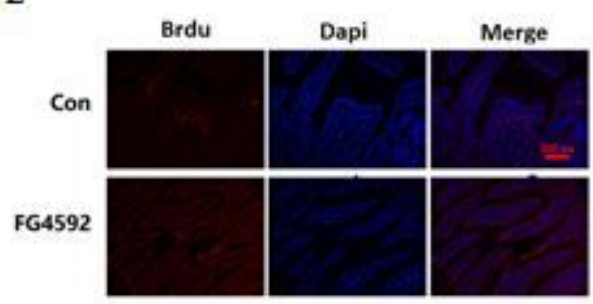

B

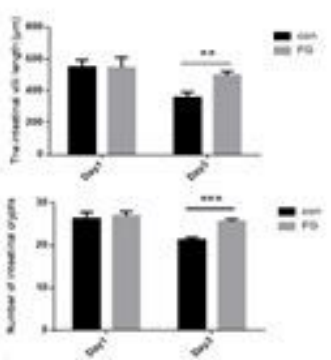

D

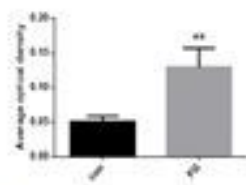

$\mathbf{F}$

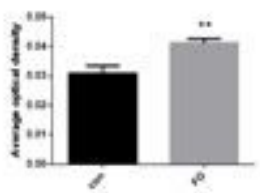

\section{Figure 2}

FG4592 remits radiation-induced intestinal injury of mice. 2 hours before radiation the mice were intraperitoneal injected with FG4592 $(25 \mathrm{mg} / \mathrm{kg})$, and then received abdominal radiation of 25Gy. At day day 1 and day 3 the small intestine tissues were taken to perform H\&E staining $(A)$, with which the length of intestinal villi and the number of crypts were also quantified (B). The Lgr5+ fluorescence staining was used to assess the amount of intestinal stem cells (C), and Brdu fluorescence staining could determine the proliferation of intestinal epithelial cell (E). (D) and (F) were quantifications for Lgr5+ fluorescence and Brdu fluorescence, respectively. The statistical results are expressed by Mean $\pm S D$. ** represents $p$ $<0.01$, *** represents $p<0.001$. 

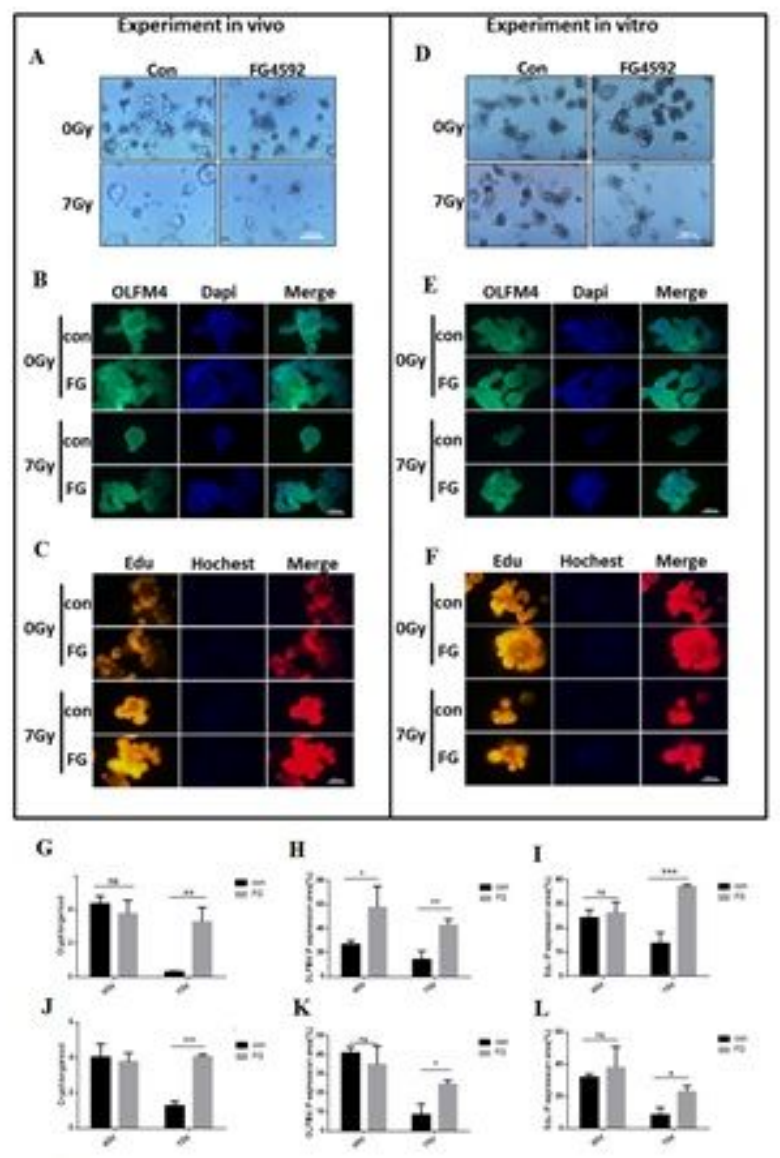

M

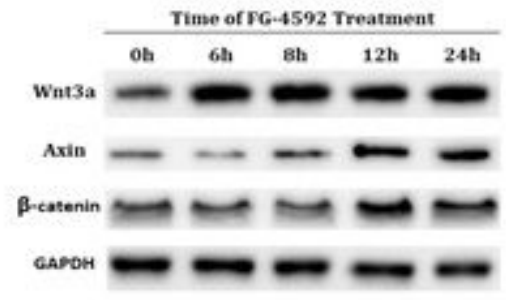

Figure 3

FG4592 promotes the regeneration and differentiation of intestinal stem cells by activating Wnt/ $\beta$-catenin pathway. Experiments in vivo: Mice were given $25 \mathrm{mg} / \mathrm{kg}$ of FG4592 by intraperitoneal injection and then received $25 \mathrm{~Gy}$ abdominal radiation $(1 \mathrm{~Gy} / \mathrm{min}) .24$ hours after radiation, the small intestinal crypts were extracted to culture organoids. 5 days later, the morphology of organoid was observed (A) and OLFM staining were conducted to mark small intestinal stem cells (B). Simultaneous Brdu assay was used to test the proliferation ability of organoids $(C)$. The amounts of organoids were calculated $(G)$. $(H)$ and $(I)$ were the quantification for OLFM staining and Brdu assay, respectively. Experiments in vitro: The

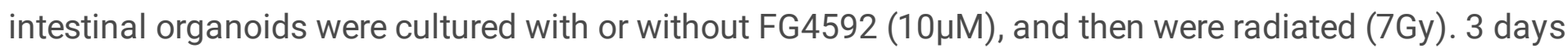
later, the morphology of organoid was observed (D), amounts of organoids were calculated $(\mathrm{J})$, and fluorescence intensity of OLFM staining $(E, K)$ and $\operatorname{Brdu}(F, L)$ were tested. Moreover, at different time points $(6,8,12,24 \mathrm{~h})$ after FG4592 $(10 \mu \mathrm{M})$ treatment, the expression of Wnt3a, Axin and $\beta$-catenin were 
detected by Western blotting (M). The statistical results are expressed by Mean $\pm S D$. * represents $p<0.05$, ** represents $p<0.01$, ***represents $p<0.001$.

A

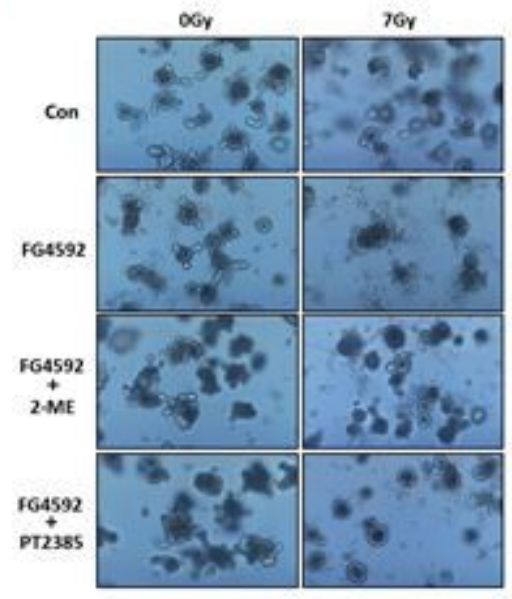

B

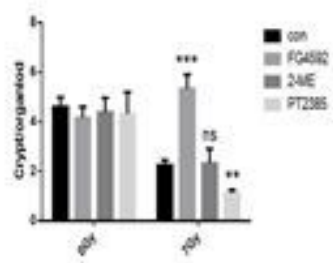

C

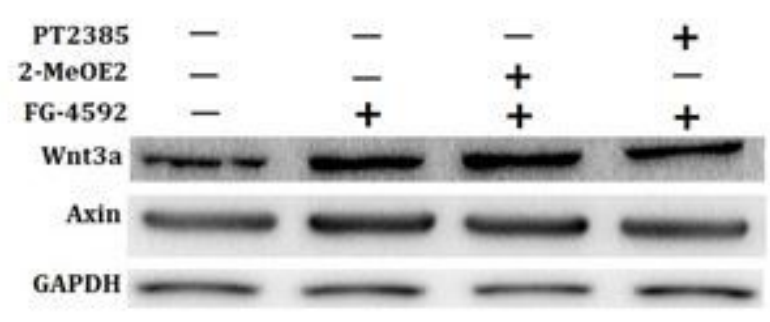

\section{Figure 4}

FG4592 protects against radiation-induced intestinal injury by up-regulating HIF-2 rather than HIF-1. Small intestinal organoids were treated with FG4592 for 2 hours, and simultaneously the HIF-1 inhibitor, 2-Methoxyestradiol (2-MeOE2) and HIF-2 inhibitor, PT2385 were added. 3 days after receiving 7Gy radiation, the morphology of organoids was observed $(A)$ and amounts were calculated (B). Simultaneously the proteins of organoids were extracted to detect the expression of Wnt3a and Axin by Western Blotting (C). The statistical results are expressed by Mean \pm SD. * represents $p<0.05$, ** represents $p<0.01$, *** represents $p<0.001$. 
A

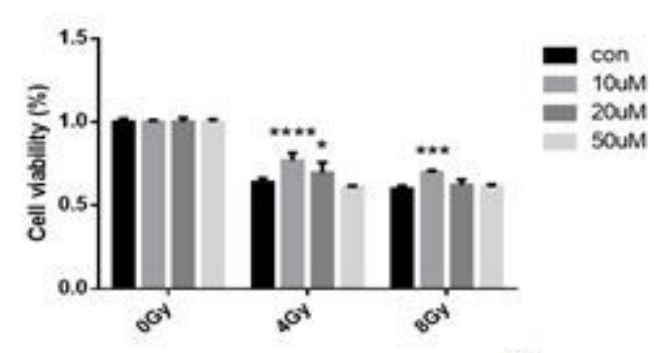

B

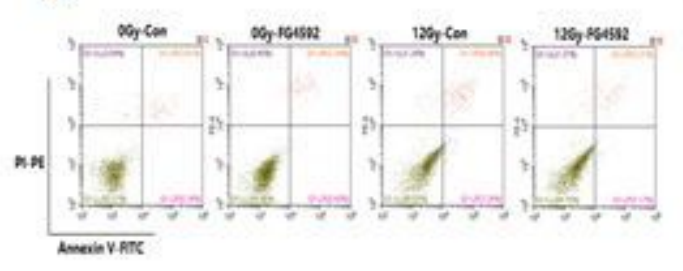

D

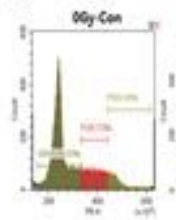

F
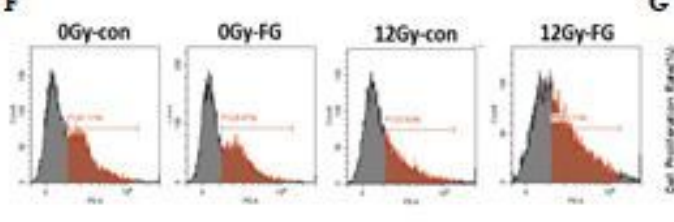

c

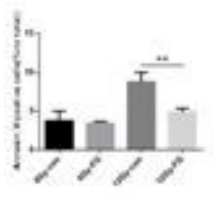

E

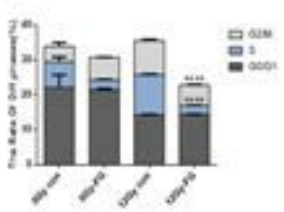

G

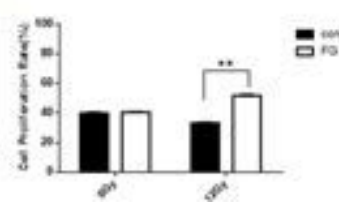

Figure 5

FG4592 reduces damage of intestinal epithelial cells from radiation. Modek cells were pretreat with or without FG4592 $(10 \mu \mathrm{M})$, and then received 12Gy radiation. 24 hours later, the cell viability was detected by CCK-8 assay (A). By flow cytometry the apoptosis was determined at 48 hours (B, C) and cell cycle was tested at 8 hours (D, E) post-radiation. Brdu assay was used to assess proliferation of Modek. 48 hours after radiation, the immunofluorescence of Brdu was examined by flow cytometry $(F)$ and fluorescence intensity was quantitated $(G)$. The statistical results are expressed by Mean $\pm S D$. * represents $p<0.05$, ** represents $p<0.01$. 


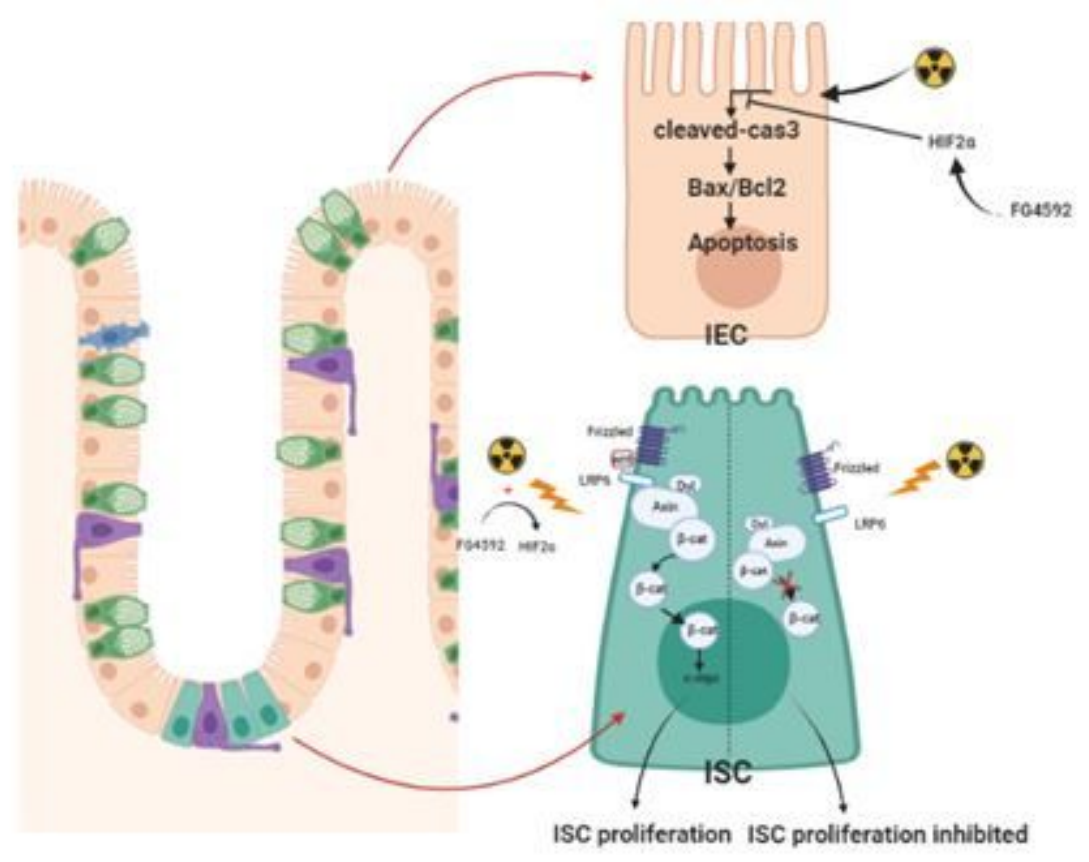

Figure 6

FG4592 reduces damage of intestinal epithelial cells from radiation. Modek cells were pretreat with or without FG4592 $(10 \mu \mathrm{M})$, and then received 12Gy radiation. 24 hours later, the cell viability was detected by CCK-8 assay (A). By flow cytometry the apoptosis was determined at 48 hours (B, C) and cell cycle was tested at 8 hours (D, E) post-radiation. Brdu assay was used to assess proliferation of Modek. 48 hours after radiation, the immunofluorescence of Brdu was examined by flow cytometry $(F)$ and fluorescence intensity was quantitated $(G)$. The statistical results are expressed by Mean $\pm S D$. * represents $p<0.05$, $\star \star$ represents $p<0.01$. 